\title{
El concepto de estrategia y la Nueva Etnohistoria
}

\section{The concept of strategy and the Nueva Etnohistoria}

\author{
Alejandra Ramos ${ }^{1}$ http://orcid.org/0000-0003-1373-0523 \\ Carlos María Chiappe ${ }^{2}$ http://orcid.org/0000-0002-1760-6004 \\ ${ }^{1}$ Universidad de Buenos Aires - Consejo Nacional de Investigaciones Científicas y Técnicas, \\ ARGENTINA. Email: alejandraramos_@hotmail.com \\ ${ }^{2}$ Instituto de Investigaciones Arqueológicas y Museo R. P. Gustavo Le Paige s. j., \\ Universidad Católica del Norte, CHILE. Email: carlos.chiappe@ucn.cl
}

\section{Resumen}

En este artículo realizamos una revisión del uso del concepto de estrategia en las ciencias sociales, en particular en el campo de la etnohistoria andina. Nos centramos en la fase denominada Nueva Etnohistoria (1980 en adelante), momento de consolidación transcontinental de este campo interdisciplinar. Las fuentes de información empleadas consisten en publicaciones colectivas de relevancia y documentos inéditos relativos a la organización del Primer Congreso Internacional de Etnohistoria. Con base en ellas identificamos los sentidos asociados al concepto de estrategia y reconstruimos los debates que su aplicación suscitó, los cuales están ligados a problemáticas científicas vigentes. A partir de este recorrido fundamentamos la necesidad de reactualizar la revisión crítica del concepto bajo análisis.

Palabras claves: etnohistoria, Andes, estrategia, estudios sobre ideas.

\begin{abstract}
In this article we review the use of the concept of strategy in the social sciences in particular in the field of Andean Ethnohistory. We focus on the phase called Nueva Etnohistoria (1980 and later), a moment of transcontinental consolidation of this interdisciplinary field. The sources of information used in this study consist of relevant collective publications and unpublished documents related to the organization of the I International Congress of Ethnohistory. With respect to these, we identify the meanings associated with the concept of strategy and reconstruct the debates that arise in its application, which are linked to current problems in the social sciences. Based on the foregoing, we propose the need to update the critical review of the concept of strategy.
\end{abstract}

Keywords: ethnohistory, Andes, strategy, studies on ideas.

Recibido: 5 septiembre 2018. Aceptado: 6 diciembre 2019 


\section{Introducción}

El uso del concepto de estrategia se encuentra ampliamente extendido en múltiples campos y disciplinas. Tras un empleo inicial asociado al campo militar y a la teoría de la praxis marxista, en la década del cuarenta se vinculó fuertemente a la teoría de los juegos. Esta estuvo en sus inicios concebida para contribuir a la comprensión de la economía, con la ya clásica publicación Theory of Games and Economic Behavior (Von Neumann y Morgenstern, 2007 [1944]). La estrategia era entendida como la elección que realiza un jugador entre un conjunto de opciones posibles y en un entorno determinado, donde el resultado depende no solo de sus propias acciones sino de la acción de otros. Durante la década del cincuenta, en plena Guerra Fría, se delinearon los postulados básicos de la teoría de los juegos y su aplicación se extendió a la filosofía y la ciencia política.

El concepto de estrategia amplió sus fronteras en los años sesenta extendiéndose a la gestión de empresas y a los planes estatales y de organismos internacionales de desarrollo. En los años setenta entró en boga en la biología, de la mano de otros conceptos como supervivencia y adaptación, mientras que en los ochenta se instaló en la pedagogía, tanto en términos de estrategias de enseñanza como de aprendizaje (Berry, 1967; Ridley, 1993; Monereo, 1999).

En el campo de los estudios andinos, su boom coincidió con el auge de la Nueva Etnohistoria. Si bien puede rastrearse alguna definición de etnohistoria hasta la primeras décadas de siglo XX, lo cierto es que su desarrollo es propio de los tiempos de la segunda posguerra y del proceso de descolonización concomitante. En la región andina, ${ }^{1}$ y particularmente en Perú, fue en las décadas del sesenta y setenta que la etnohistoria se instituyó (Ramos, 2016a, 2016b). En los años ochenta, tal como veremos luego con mayor detalle, la Nueva Etnohistoria recuperó el enfoque interdisciplinario y la apuesta metodológica

1 La idea de región andina y la adjetivación de la etnohistoria como andina no son categorizaciones transparentes. Para un examen de los sentidos que adquiere lo andino y su relación con la etnohistoria remitimos a Ramos (2018). de recurrir a diversas y renovadas fuentes de información, hecho impulsado ya en las décadas precedentes. A la vez, se enfatizó la necesidad de superar las visiones dicotómicas y focalizar en los entramados de sistemas que se produjeron durante la Colonia y la República (Chiappe, 2018). Es justamente desde ese interés por las distintas fuerzas que limitan o influyen a otras que el concepto de estrategia se tornó omnipresente en la Nueva Etnohistoria.

La denominación de Nueva Etnohistoria se corresponde con la década del ochenta. Posteriormente, el impacto de las críticas de raíz posmoderna en las ciencias sociales en general y en la etnohistoria en particular delinearon otros caminos de conceptualización y definición de las prácticas académicas (Ramos, 2016a). En el marco de estas coordenadas de espacio y tiempo -los Andes en los ochenta-, y teniendo en cuenta la importancia de las condiciones materiales y del lenguaje en la producción de las ideas (Consejo de Dirección Prismas, 2007), nos preguntamos en este artículo cómo fue empleado el concepto de estrategia. Para abordar este interrogante nos valdremos de publicaciones académicas y de material en gran parte inédito relativo a la organización del I Congreso Internacional de Etnohistoria (I CIE) realizado en 1989 (Ramos y Chiappe, 2018).

Nuestro corpus está conformado por tres compilaciones que consideramos representativas de la Nueva Etnohistoria (Ramos, 2016a; Chiappe, 2018) y por la correspondencia entre organizadores, coordinadores y asistentes al primer CIE. Las compilaciones son producto de la misma iniciativa, impulsada por referentes del campo, y reúnen varias decenas de artículos. La correspondencia cubre los dos años que tomó la organización del primer CIE y está compuesta por más de 250 documentos.

El tipo de material seleccionado nos permitirá introducir una reflexión sobre la complementariedad de las fuentes. Es decir, cómo la información contenida en textos publicados puede ser ampliada a través de la revisión del intercambio epistolar, entre otras fuentes inéditas. El análisis de las fuentes de información que conforman nuestro corpus (obras colectivas publicadas y correspondencia inédita) se realizará atendiendo a los contextos en que aparece mencionado el concepto (a qué temas, categorías y 
sujetos es asociado), qué sentidos le son asignados por los distintos investigadores pertenecientes al campo etnohistórico y si estos son complementarios o no.

Para abordar nuestro objetivo de reconstruir los sentidos y alcances del concepto de estrategia en la Nueva Etnohistoria, primeramente referimos su aparición en las ciencias sociales, lo que nos permitirá situar la especificidad del debate en la etnohistoria. Seguidamente, contextualizamos el momento del surgimiento y consolidación de la etnohistoria andina (década del setenta) para luego detenernos en la Nueva Etnohistoria (década del ochenta). Posteriormente, rastreamos el empleo del concepto de estrategias en tres compilaciones centrales del período. Luego, abordaremos las discusiones al respecto en el marco de la organización del primer CIE, que tuvo lugar en Buenos Aires en 1989. Finalmente, evaluamos la complementariedad de las fuentes seleccionadas y los beneficios de una aproximación metodológica de este tipo para el estudio del desarrollo de los campos disciplinares.

\section{El concepto de estrategias en las ciencias sociales}

Para el caso de la etnohistoria o -más ampliamentelo que se denomina historia indígena (sensu Hidalgo Lehuedé, 2004), se ha señalado que estudios de caso como los referidos al liderazgo étnico andino fueron encarados desde mediados de la década del sesenta desde la perspectiva de las estrategias por los sujetos involucrados, consolidándose esto en las dos décadas posteriores (cfr. Morrone, 2007).

Lo anterior debe ser incluido en el plano general del desarrollo de las ciencias sociales de la época. En efecto, a partir de la década del setenta, a raíz de una preocupación creciente por demostrar la capacidad de agencia de una pluralidad de sujetos hoy comúnmente denominados subalternos, los cientistas sociales comenzaron a utilizar asiduamente el concepto de estrategias.

Más allá de algunos casos de autores enfocados en acciones y elecciones individuales, el término estrategias en las ciencias sociales fue generalmente utilizado para dar cuenta de prácticas colectivas que se despliegan en el tiempo, que conforman cierto patrón que no es producto del azar y que pueden ser tanto conscientes como inconscientes, aunque esto último ha sido una de sus características más debatidas. Un autor emblemático, tanto en relación con la delimitación del concepto como en cuanto a la pluralidad de campos desde los cuales fue retomado, es Pierre Bourdieu (1972). En su obra, las estrategias son concebidas como un mecanismo de reproducción social que incorpora un "conjunto de prácticas fenomenalmente muy diferentes" instrumentadas por los agentes "de manera consciente o inconsciente" para "mantener o mejorar su posición en la estructura de las relaciones de clase" (Bourdieu, 1988).

Puede decirse que el uso del concepto de estrategia que por entonces hacían cientistas sociales latinoamericanos -entre ellos los andinólogos- estaba a tono con las prácticas de reproducción enfocadas por Bourdieu (1988). Señala Alicia Gutiérrez (2002) que, en los análisis de los fenómenos de la pobreza urbana, se buscaba entender los mecanismos que posibilitan la reproducción social a pesar de las fuertes restricciones estructurales. El concepto de estrategias permitía mantener la equidistancia entre el accionar de los sujetos y la limitación de las estructuras, reservándose "un margen de opción a los agentes sociales, con lo que sus estrategias no están completamente determinadas por factores estructurales ni son el mero resultado de una libre elección individual" (Gutiérrez, 2002, pp. 13-17).

La aplicación del concepto por parte de diferentes investigadores llevó a buscar mayor precisión en el universo de referencia mediante el agregado de términos como "de supervivencia", "de reproducción", "de existencia", "de resistencia", "familiares" y "adaptativas", entre otros. Empero, esto no hizo más que replicar la dificultad inicial, tal como destacó coetáneamente Argüello (1981, p. 191):

Con el concepto de 'estrategias de supervivencia' parece estar ocurriendo [que,] frente a una rápida y generosa difusión de este preconcepto, se busca utilizarlo para cubrir nuevas y diferentes áreas de la realidad social, asignándole cada vez nuevos contenidos 
y haciendo peligrar con ello la identificación y tratamiento de un fenómeno específico que afecta a un grupo determinado de la sociedad y que plantea un tema científico y socialmente relevante.

Cuatro décadas después, a pesar del empleo extendido del concepto, Viazzo y Lynch (2002, p. 425) consideran que este permanece ambiguo y mal definido:

We can legitimately wonder whether we are dealing here with a well-developed concept or just with a fashionable and rather equivocal term. A certain amount of polysemy can of course be expected when a term is used as is the case with 'strategy'- across the social sciences, from economics to sociobiology. However, even if we restrict our attention to family history and to social anthropology, we immediately notice an alarming degree of looseness and confusion.

Más allá de las limitaciones señaladas, el concepto de estrategias fue efectivo porque, 1) logró captar a investigadores provenientes de distintas $-e$ incluso opuestas- corrientes teóricas y, 2) permitió recuperar simultáneamente las experiencias históricamente acumuladas y la capacidad de innovación social (Sánchez Parga, 1984). Ciertos temas y campos giraron mayoritariamente en torno al mismo y fueron redimensionados en parte a partir de su implementación, como los estudios sobre migración, reciprocidad, mercantiles y parentesco, entre otros. Así visto, no es casual que el concepto de estrategia se retomara rápida y ampliamente en la etnohistoria, campo en el que estas temáticas ocupaban un lugar central.

La Nueva Etnohistoria se construyó en el diálogo entre la historia, la arqueología y la antropología social (Chiappe, 2018; Ramos, 2016a). En este sentido, es de interés considerar la definición de Kirch (1980, p. 129) de las estrategias adaptativas como

[...] the set of culturally transmitted behaviors (extractive, exploitative, modifying, competitive, mutualistic, and the like) with which a population interacts or interfaces with its natural and social environment interacts or interfaces with its natural and social environment.

Particularmente desde el paradigma procesualista el concepto referido tenía por entonces fuerte incidencia en la arqueología, y encontraremos su eco en algunas posiciones de los etnohistoriadores.

\section{La etnohistoria andina antes de la Nueva Etnohistoria}

El término etnohistoria comenzó a ser profusamente empleado a mediados del siglo XX. A través de él se designó una articulación entre Antropología e Historia que el mundo de posguerra, en pleno proceso de descolonización, parecía demandar. Surgida de este clima común, la articulación disciplinar estuvo anclada en contextos nacionales y continentales, y se vio condicionada por el tipo de materiales con los que se trabajaba y los principales agentes involucrados. La etnohistoria mexicana y la peruana compartieron el anclaje en grandes civilizaciones precolombinas, pero el hecho de que la primera contara con textos en náhuatl, maya, yucateco, zapoteco y otomí, entre otras lenguas, fue uno de los principales elementos de diferenciación entre ambas (Ramos, 2016b, pp. 15-34).

El boom de la etnohistoria andina se produjo a fines de los ańos sesenta y durante la década del setenta, como parte de una historia de los pueblos sin escritura. Esta promovía la articulación de distintas disciplinas, acompañado de "un cambio de actitud en el estudio de las sociedades andinas" (Pease, 1987 [1974], p. 177) que comenzaban ahora a incluirse en las historias nacionales. John Murra fue uno de sus grandes propulsores, caracterizándose su accionar por el aliento a los investigadores noveles, la formación de instituciones, su modelo teórico de verticalidad y su propuesta de construcción de conocimiento interdisciplinar (Chiappe, 2018). ${ }^{2}$

2 Respecto de John Murra y su rol en la conformación de una comunidad andina de investigadores, puede verse Ramos (2015). 
Este momento de expansión se destacó por: 1) los nuevos anclajes institucionales que, excediendo el Perú, surgieron en Bolivia, Argentina, Chile, Ecuador y Colombia; 2) las fuentes inéditas, localizadas mediante el trabajo de archivo (p.e. documentos judiciales y eclesiásticos, visitas y testamentos), novedosas en relación con aquellas que se venían utilizando (como crónicas de conquista y relatos de viajeros), las cuales presentaban un mayor sesgo eurocéntrico y cuscocéntrico; 3) la publicación o reedición en versiones mejoradas y/o de mayor distribución de los documentos; 4) el abordaje interdisciplinario entre historia, antropología y arqueología; 5) los primeros balances y propuestas a futuro; 6) la reflexión sobre su objeto, disciplina y método; y 7) las nuevas síntesis y modelos teóricos (Chiappe, 2018).

Paralelamente, el campo académico-científico latinoamericano sufría una acelerada expansión desde mediados del siglo XX, la que se sostuvo a lo largo de las siguientes décadas, con las luces y sombras derivadas de los fenómenos de transformación y reversión social que experimentaron nuestros países. Durante la década del setenta las redes académicas transnacionales siguieron ampliándose, los estudios etnohistóricos se expandieron y se consolidaron nuevos medios de comunicación, como congresos y publicaciones periódicas.

En esta misma década fue clave el surgimiento o la reestructuración de revistas (p.e. Chungara, Revista del Museo Nacional (Perú), Historia y cultura, Pumapunku, Bulletin IFEA, Estudios Atacameños, Allpanchis) en donde se publicó buena parte de la producción etnohistórica de aquellos años. También tuvieron lugar una serie de encuentros académicos (Seminario sobre Reinos Lacustres, primer Congreso del Hombre Andino, Programa Especial de Estudios "Otońo Andino", Congresos Peruanos del Hombre y la Cultura Andina, Jornadas de Etnohistoria del Museo Nacional (Perú), Coloquio "Críticas y perspectivas de la Arqueología Andina") que sirvieron para acordar definiciones en relación con los principales conceptos de este campo.

A nivel teórico-metodológico, el gran avance de esta década consistió en que la articulación entre Antropología e Historia habilitó la construcción de una historia andina, lo que estuvo -como apuntamos al comienzo de este apartado- atravesado por el contexto poscolonial. Como señaló oportunamente Nathan Wachtel (1976 [1971]):

¿Es acaso un azar que la historiografía referida a América Latina se centre sobre todo en la civilización española, mientras que la historia del mundo indígena, desde la Conquista hasta nuestros días, permanezca, por así decirlo, desconocida? Es preciso esperar a los tiempos actuales, al fin de la hegemonía europea y a los movimientos de descolonización, para que Occidente tenga conciencia de que también existen otras sociedades, es decir, que tienen su historia particular y de que ésta no sigue necesariamente las huellas del modelo europeo (p. 23).

En efecto, la Visión de los vencidos, obra representativa de la época, puso foco en la historia de larga duración de las sociedades andinas conquistadas por medio de una mirada en tres niveles: "el de los acontecimientos (la Conquista en sentido estricto, vista por los indios), de las estructuras (las de la sociedad peruana, después de la Conquista en un sentido amplio) y de la praxis (en un caso privilegiado, el de las rebeliones indígenas)" (Wachtel, 1976 [1971], p. 34). Es en este sentido que Pease (1998, p. 14) afirmó que las investigaciones etnohistóricas de la década del setenta posibilitaron concebir una historia andina que hasta 1960 había resultado "impensable".

La propuesta de Pease nos remite a las consideraciones hechas por Bourdieu (2007 [1980]) sobre las ciencias sociales coloniales. Lo impensable de una época es aquello que no se puede intelectualizar "por falta de disposiciones éticas o políticas que inclinen a tomarlo en cuenta y en consideración" y también "de instrumentos tales como problemáticas, conceptos, métodos y técnicas" (Bourdieu, 2007 [1980], p. 16). Hasta la década del sesenta se había puesto énfasis - por una parte- en los aspectos de clase (campesinado) en detrimento de los étnicos y -por otra- recién estaba comenzando a implementarse el aparato teórico-metodológico que permitió luego dar cuenta de la historicidad de las 
sociedades no-occidentales. Por lo anterior podría plantearse que la empresa intelectual de realizar una historia andina era, hasta ese momento, virtualmente impracticable.

Aunque en la década del setenta se hizo foco en la desestructuración social que sufrieron los colectivos indígenas -lo que luego sería ampliamente discutido-,${ }^{3}$ el gran logro de las investigaciones de estos ańos fue la producción de un conocimiento centrado en una visión andina de la historia. Un momento superador se iniciará en la década siguiente, cuando el foco de las investigaciones se traslade a la agencia indígena.

\section{Hacia una nueva etnohistoria}

La década del ochenta marcó la proliferación de las líneas de investigación vinculadas a la Nueva Etnohistoria. Sobre la base de los desarrollos de la década anterior, como el trabajo interdisciplinar y el uso de fuentes documentales de nuevo tipo abordadas con mirada antropológica, esta se caracterizó por la discusión de modelos teóricos, una impronta regional en las investigaciones y la focalización en los entramados de sistemas que se produjeron durante la Colonia. Dentro de esta corriente, y en particular en esta década, el concepto de estrategias en tanto práctica social fue central (Martínez Cereceda, com. pers., 2017). Esto puede verse a través de los diversos medios de difusión del conocimiento etnohistórico de la época, como obras colectivas y eventos académicos. En este apartado bosquejaremos la aparición del concepto de estrategias en tres conferencias realizadas en 1986 en Sucre, Madison y Quito bajo el impulso del Social Science Research Council (SSRC) y el American Council of Learned Societies (ACLS).

En 1981 el Joint Committee on Latin American Studies del SSRC y el ACLS auspiciaron una reunión coordinada por Brooke Larson y Steve Stern de la que participaron Carlos Sempat Assadourian,

3 Considérese la diferencia de énfasis que resulta de la comparación de los títulos La vision des vaincus de Nathan Wachtel, publicado en 1971, y Oprimidos pero no vencidos, de Silvia Rivera Cusicanqui, publicado en 1984, así como la década que los separa.
José María Caballero, Magnus Mörner, John Murra, Silvia Rivera Cusicanqui, Karen Spalding y Enrique Tandeter. El propósito era diseñar un proyecto sobre la historia andina, por lo que se programaron tres conferencias temáticas cuyo fin fue discutir los avances más recientes en las investigaciones sobre historia y etnología y sentar las bases para los estudios futuros. Estas compartían un doble objetivo "1. analizar las diversas formas en que los pueblos andinos han participado en y respondido $a$ los mundos sociales, económicos, culturales y políticos en que se desenvolvieron, y 2 . buscar indagar las motivaciones y las dinámicas endógenas de la experiencia histórica andina" (Stern, 1990b [1987], p. 14).

\section{Mercados en Sucre}

La primera conferencia se desarrolló en Sucre en 1983 bajo el nombre "Penetración y expansión del mercado en los Andes, siglos XVI-XX". Coordinada por Olivia Harris, Brooke Larson y Enrique Tandeter, tuvo por objetivo atender, siguiendo una línea antropológica, la durabilidad y el impacto de los sistemas económicos indígenas en la expansión mercantil. Los trabajos allí presentados fueron publicados en La participación indigena en los mercados surandinos (Harris, Larson y Tandeter, 1987).

El prólogo a la primera compilación presentó el proyecto de las tres reuniones como parte de una "nueva etnohistoria" (Harris et al., 1987). En la Introducción se manifestaba que uno de los objetivos del volumen era salvar la distancia tradicional entre la insistencia de los etnohistoriadores en la durabilidad de las normas culturales e instituciones andinas y el análisis de los historiadores económicos, que focalizaban en el poder de transformación del mercado y el Estado durante los períodos colonial y republicano (Harris et al., 1987, p. 21). Se proponía así romper con el dualismo entre lo andino y lo mercantil y ganar precisión regional y temporal.

La noción de estrategias se volvía central tanto porque permitía discutir la visión de "una prolongada y unívoca historia de resistencia a las fuerzas coactivas y/o libres del mercado" como también recuperar "la perspectiva de los actores andinos". El foco estaba puesto en analizar las "estrategias andinas que 
se mueven en los márgenes que las fuerzas globales permiten, pero que, a su vez, ponen límites a esas fuerzas e influyen sobre ellas (Harris et al., 1987, pp. 39 y 41).

Para evidenciar el rol central del concepto de estrategias para el estudio de las sociedades andinas en la compilación de Harris et al. (1987), tomaremos dos ejemplos. El primero, un aporte de Thierry Saignes (1987) sobre las migraciones andinas en el área de influencia de Potosí en el siglo XVII. El segundo, un texto de Jorge Dandler (1987) sobre el fenómeno de diversificación económica familiar en Cochabamba. Es decir, dos trabajos sobre problemáticas andinas con marcos temporales muy distantes entre sí (tres centurias) y fuentes de información diversas (predominantemente documentales en el primero y etnográficas en el segundo).

Saignes (1987) postula que las migraciones internas, en tanto 'reajustes socio-étnicos postoledanos', fueron una 'respuesta andina' a un escenario caracterizado por el abandono de las reducciones indígenas y el quiebre del trabajo forzado en las minas. Si bien, para algunos autores, ese contexto evidenciaba un fenómeno de 'ruptura o desinscripción étnica', el autor plantea, en cambio, que el reasentamiento generalizado de la población indígena habría correspondido a "estrategias originales frente al asedio colonial”. Para probarlo, va a focalizar en el accionar de caciques y migrantes mediante el estudio de tres indicadores: 1) los antiguos y los nuevos caminos de acceso a los recursos de diferentes pisos ecológicos; 2) las estrategias de los kurakas frente a las presiones de los españoles; y 3) las "modalidades de inscripción de los migrantes con respecto a sus comunidades de origen y de acogida" (Saignes, 1987, pp. 112-113).

El estudio de las fuentes permitió sostener que las migraciones internas, que abarcaron tanto movimientos interecológicos como interregionales, no fueron aleatorias, ya que se vieron influenciadas por la expansión y la retracción de la economía minera. Y, más importante aún, que no fueron -en sí mismas- disgregadoras o aglutinadoras de los distintos agrupamientos sociales. Además, en muchos casos, posibilitaron el aumento de estatus individuales (Saignes, 1987, pp. 143-144). Por su intermedio,
El mundo andino [...] logró adaptarse a las nuevas realidades coloniales: durante el siglo XVII maneja a la vez un ideal adscriptivo, propio de la sociedad 'holista' prehispánica (cualquier individuo está subordinado a un ayllu) y un deseo, vehiculizado por los valores feudales y mercantiles europeos, de salvación y ascenso personal. Estos dos ideales no se excluyeron mutuamente: el éxito individual necesita recibir su confirmación social y cósmica; la identidad colectiva necesita definirse con respecto a un lugar (Saignes, 1987, p. 144).

En el escenario descrito, los miembros dispersos de un ayllu siguieron perteneciendo a él, mientras que los extranjeros pudieron incorporarse a un ayllu diferente al de su origen porque el parentesco andino otorgaba gran valor a la alianza. En este movimiento poblacional, los caciques y los migrantes competían y a la vez cooperaban estratégicamente en orden de aprovechar las nuevas coyunturas económicas "buscando [...] un nuevo lugar en el ámbito colonial [y] nuevos lazos contractuales dentro de la racionalidad andina" (Saignes, 1987, p. 146).

En el caso de Dandler (1987), teniendo también como trasfondo el interés por los procesos de cambio social, el foco está puesto en analizar las formas de inserción de las economías campesinas en la economía capitalista. El autor discute las posturas académicas que proponían que la inserción del campesinado andino en el mercado capitalista llevó a su descampesinización, proletarización o reestructuración. Aunque en otro escenario histórico, nos encontramos así en un terreno similar a la visión negativa de Saignes (1987) sobre las supuestas rupturas y desinscripciones étnicas del siglo XVII.

Los argumentos sobre la proletarización del campesinado andino planteaban que el estado precario del mismo se debía a la extrema parcelación de las tierras, la consecuente insuficiencia productiva y $-\mathrm{a}$ la postre- la necesidad de vender la fuerza de trabajo en actividades extraagrícolas para completar la renta familiar (Dandler, 1987). Otras posturas, sin embargo, enfatizaban "las formas persistentes de organización campesina y su capacidad de resistencia 
frente a los mecanismos del mercado y otras fuerzas económicas" (Dandler, 1987, p. 641). Estas, si bien reconocían la tendencia general hacia la diversificación en las actividades no agrícolas, hacían énfasis en que la producción agrícola seguía siendo el eje de la economía campesina. Se precisaba entonces desarrollar una perspectiva analítica que permitiera advertir los matices del fenómeno de proletarizacióndescomposición (Dandler, 1987, p. 645). El autor la encontrará mediante la adopción de la herramienta conceptual "estrategia de vida". Comentando la investigación en Cochabamba, se plantea que un aspecto central de la misma fue el estudio de

\section{[...] la diversificación económica y su rol en} las estrategias de vida de unidades domésticas campesinas [...] la noción de estrategia de vida incluye la idea de que no sólo es necesario contar con recursos como tierra, capital y fuerza de trabajo, sino también con elementos menos tangibles como tiempo, información, redes sociales e identidad, para visualizar oportunidades, incluso en situaciones sumamente limitadas por un contexto económico o una gran crisis de la economía nacional [...]. La idea de estrategia de vida, por lo tanto, admite una estrategia analítica más amplia sobre un fenómeno económico y social dinámico, como por ejemplo la necesidad de las familias campesinas de mantener arraigo en su aldea o localidad, donde ya no es posible residir sin desarrollar dentro y fuera de ella una gama de actividades especializadas y diversas. Esto implica una movilización familiar intensa y constante que inclusive se expresa espacialmente a nivel cotidiano como una estrategia para ampliar el acceso a recursos económicos (Dandler, 1987: 652-653).

La diversificación observada implicaba una "variedad de formas y contenidos", entre los cuales se contaban tanto las estrategias "de unidades domésticas pauperizadas que se dedican a diversas y fluctuantes actividades económicas" como la "ampliación de la base de recursos y especialidades que permiten a la unidad doméstica un cierto grado de capitalización" (Dandler, 1987, p. 682).
En definitiva, los efectos de fenómenos como las migraciones internas de los forasteros del siglo XVII (Saignes, 1987) o la diversificación económica de las unidades familiares cochabambinas del siglo XX (Dandler, 1987) no llevaban inequívocamente a la desinscripción étnica (en el primer caso) o a la proletarización (en el segundo caso). Y, aunque muchas veces el resultado pudiera ser ese, era central empezar a analizar e interpretar estos fenómenos mediante un enfoque que permitiese observar las estrategias individuales y/o colectivas frente a las fuerzas mercantiles. Estas habían permitido -y permitían hoy día- aprovechar los complejos escenarios de las crisis económicas en el marco de las cuales las investigaciones de ambos autores se enmarcan: el Virreinato del Perú del siglo XVII y la Bolivia de la década de 1980.

\section{Resistencias en Madison}

La segunda conferencia estuvo a cargo de Steve Stern, llevó el nombre de "Resistencia, rebelión y conciencia campesina en los Andes, siglos XVIII al XX" y fue realizada en Madison en 1984. Aunque no siempre explícitamente, el concepto de estrategias se halla en el corazón de las ideas expresadas en la compilación, específicamente en el intento de recuperar el mundo cultural y las luchas de los actores subalternos, reparando en la relación que establecieron con las instituciones dominantes. Tal como propone Stern en la Introducción a la obra colectiva producto del encuentro, se buscaba ofrecer una 'explicación alternativa respecto a la insurrección en el período colonial tardío', y para ello proponía: 1) devolver a los campesinos el papel de sujetos activos e iniciadores de relaciones políticas; 2) seleccionar marcos temporales de larga duración, para observar la evolución de los patrones preexistentes de adaptación en resistencia que los grupos subalternos desarrollaron en relación con una estructura de dominación política; 3) tomar en cuenta la heterogeneidad de la conciencia y los horizontes políticos campesinos y 4) realzar la importancia de la etnicidad en las revueltas campesinas latinoamericanas (Stern, 1990c [1987], pp. 32 y 36).

En su artículo "La era de la insurrección andina, 1742-1782: una reinterpretación”, Stern plantea 
que, en la primera mitad del siglo XVIII "la cambiante economía política de la explotación mercantil había socavado las anteriores estrategias y relaciones del gobierno colonial y de resistencia andina, virtualmente a todo lo largo de la sierra peruana y boliviana" (Stern, 1990d [1987], p. 92). En este marco, las rebeliones aparecen como una innovación en las estrategias frente a las nuevas condiciones sociales.

Sin embargo, las implicancias del concepto de estrategias en esta compilación tienen un alcance mayor que el entender los levantamientos dentro de un conjunto de prácticas posibles. Esto puede apreciarse cuando se hace referencia a la necesidad de comprender el "contexto social e ideológico" de los movimientos sociales andinos (Campbell, 1990 [1987], p. 138). La situación de partida, las características del entorno y de las relaciones con los otros, las condiciones de posibilidad, en suma, constituyen un aspecto fundamental del concepto de estrategias. Se trataba de recuperar, junto a las acciones que los sujetos y los colectivos llevan adelante, el marco en el que estas se vuelven inteligibles.

El interés por comprender estos marcos atraviesa distintos artículos de la compilación, como los trabajos de Salomon y Platt, que abordan uno el siglo XVIII y otro el XIX, indagando ambos la "base moral de las relaciones políticas interétnicas" (Salomon, 1990 [1987], p. 144). También Szemiñsky (1990 [1987]), en una línea similar, se detiene en un aspecto específico relativo a los valores morales, tratando de reconstruir la ideología andina que avaló el asesinato de españoles en el marco de la insurrección del siglo XVIII.

Esta perspectiva le permite a Platt discutir, para el caso de la rebelión de Chayanta (Potosí) durante el siglo XIX, las ideas de simple manipulación criollamestiza sobre los indígenas. En términos del autor:

Son pocos los estudios de la resistencia andina que han intentado distinguir, por ejemplo, entre las instituciones y los sistemas representativos propios de cada grupo étnico o región, que hacen las veces de filtro a través del cual se perciben los diferentes cambios en la política estatal o en las corrientes culturales y económicas de amplio alcance. De ahí que surja la necesidad de integrar las perspectivas etnográficas y las evidencias documentales, a fin de poder detectar los mecanismos que rigen las alianzas o los conflictos en una región rural dominada por ayllus, como es la de Chayanta (Platt, 1990 [1987], p. 265).

En esta misma línea el texto de Albó (1990 [1987], p. 312) demuestra "que los campesinos respondieron al fracaso de los pactos paternalistas con estrategias más complejas que un simple bandazo de la política multiétnica a la independencia nativista”. El autor ve en el movimiento katarista,

[...] un intento por lograr una nueva síntesis que reemplace viejas dicotomías: una negativa a considerar clase y etnicidad como prioridades de lucha mutuamente excluyentes; una determinación de ampliar la autonomía campesina frente al Estado demandando al mismo tiempo participación en un gobierno compartido con el Estado; un esfuerzo por desarrollar una política indígena independiente, no para retraerse de la colaboración con los no-indios, sino para conquistar la ciudadanía de primera clase para negociar sobre nuevas bases la colaboración de con los nativos andinos con los trabajadores, el Estado y la nación (Albó, 1990 [1987], p. 312).

Frente a interpretaciones reduccionistas de una simple cooptación y manipulación de los indígenas por las élites nacionales, los trabajos de Madison permitieron avanzar en las nuevas circunstancias de la práctica política indígena: el hecho de la existencia de sectores criollos y mestizos dispuestos a incorporar las reivindicaciones indígenas en el corazón mismo de las naciones. Los dos polos, el multiétnico y el de autonomía indígena, en torno a los cuales oscilaron las "estrategias y conciencia política" (Albó 1990 [1987], p. 308) de las sociedades andinas en el período clásico de insurrecciones se vieron así tensionados por las nuevas posibilidades de alianza en el siglo XX. Una mirada global a esta compilación pone de manifiesto que entender el contexto simbólico que da sentido a las acciones de resistencia y 
negociación fue central tanto para repensar el ciclo de rebeliones del siglo XVIII como para reevaluar las prácticas identificadas en el siglo XX.

\section{Transformación social en Quito}

La tercera y última de las conferencias estuvo a cargo de Segundo Moreno Yánez y Frank Salomon y se realizó en Quito en 1986, llevando por título "Reproducción y transformación de las sociedades andinas, siglos XVI-XX”. Fue publicada con ese mismo nombre cinco años más tarde. El espíritu que animaba este encuentro era la convicción de que, si bien la historiografía colonial daba cuenta de las "constricciones y presiones sobre la historia nativa", era necesario complementarla con una "historiografía capaz de trabajar dentro de la realidad nativa" (Salomon, 1991, p. 9). Una propuesta de este tipo estaba orientada a pensar las fuerzas que ejercieron las organizaciones andinas y dar un paso hacia una historiografía capaz de captar -recuperando la propuesta de Sahlins- la práctica histórica de órdenes culturales diferentes. Es decir, se buscaba reconstruir una práctica histórica propiamente andina.

Por estos motivos en el encuentro se debatieron las unidades de análisis de la historiografía andina, poniendo en tensión categorías como comunidad andina, comunidad indígena, grupo étnico y nación. La historización de estas categorías y la atención a los sentidos que adquirieron en diferentes espacios y temporalidades pusieron de manifiesto que "la indefinición conceptual de los términos" se anclaba en las ambigüedades que surgían de las acciones (Salomon, 1991, p. 15). A su vez, estas eran fruto de cómo "los miembros de tales colectividades 'consumen' los términos y los refaccionan para sus propios propósitos prácticos, propósitos a veces imprevistos por los autores de la terminología impuesta. Al habilitarlos así, los contrincantes distorsionan y finalmente transforman su sentido efectivo" (Salomon, 1991, p. 15). A partir de lo anterior se remarcaba la necesidad de poner el foco en la acción, en las prácticas concretas de los grupos indígenas.

Pero, en tanto los sentidos de estas prácticas no eran transparentes, se examinaron las particularidades de las instituciones sociopolíticas al interior de la macroeconomía y de qué manera la comunicación y la acción simbólica incidieron en la solidaridad o la fragmentación de las sociedades andinas. La propuesta de fondo era que el estudio del simbolismo ofrecía una "guía para la comprensión de la acción pasada” (Salomon, 1991, p. 20) y que esta era la puerta de acceso a la comprensión de una práctica histórica andina. En este sentido, la pregunta sobre cómo los hechos económicos coloniales fueron convertidos en hechos andinos tendió un puente con la reunión de Sucre a partir del concepto de economía moral; mientras que la atención a los mecanismos que propiciaron la solidaridad/fragmentación de las unidades políticas andinas estableció una conexión con el encuentro de Madison.

El papel activo con que se invitaba a pensar a los colectivos indígenas desde la conquista del presente obligaba a analizar las prácticas particulares por medio de las cuales las agrupaciones sociales se relacionaban en un tablero de posiciones que incluía desigualdades marcadas. En este sentido, las referencias al concepto de estrategias, aunque no con un sentido unívoco, aparecen a lo largo de toda la compilación. Mientras que Abercrombie (1991) se referirá a las estrategias de subsistencia, Saignes opondrá la idea de "reajustes tácticos a las presiones coloniales" frente a la tradicional interpretación de los desplazamientos geográficos del siglo XVII como evidencia del desmembramiento de los ayllus (Saignes, 1991, p. 114). A partir de su estudio este autor propondrá que,

[...] no se puede reducir al mundo indígena a un mero "rebaño de ovejas" listos para sacrificarse a la voracidad de una "manada de lobos". La competencia entre estos últimos así como las oportunidades brindadas por el mercado colonial han dejado espacios para una amplia gama de estrategias alternativas usadas por los grupos domésticos y sus representantes (Saignes, 1991, p. 128).

Una discusión sobre el concepto aparece claramente en el aporte de Gary Urton, "Estrategias y tácticas en la historia andina”, quien plantea una diferencia en torno a los conceptos de estrategia y táctica. En efecto, siguiendo a De Certeau, Urton sostiene que estas son dos formas de actuar que 
pueden distinguirse según basen su efectividad en el control del lugar o en un manejo estratégico del tiempo, respectivamente. A partir de allí, considera que es necesario comprender "los procedimientos tácticos por medio de los cuales los individuos y los grupos domésticos han negociado sus posiciones y logrado ventajas al interior de las cambiantes relaciones de poder", "como ciertas formas de agrupaciones socio-culturales [...] cambiaban a través del tiempo en cuanto a la coordinación y movilización de nuevas tácticas", y cómo "determinadas estrategias" fueron implementadas por diferentes fuerzas dominantes, incitando a promover cambios en las agrupaciones étnicas sometidas (Urton, 1991, p. 37). En Urton, entonces, el uso de la estrategia -que parte del manejo de un determinado espacio- aparece ligado a los recursos de los dominadores, mientras que el de la táctica -entendido como el aprovechamiento del tiempo en tanto oportunidad- es herramienta de los dominados.

Lejos de menguar, como veremos en el próximo apartado, el concepto de estrategias se instalará con fuerza a las puertas del cambio de década en la discusión académica del primer Congreso Internacional de Etnohistoria.

\section{El simposio "Estrategias..." en el Primer Congreso Internacional de Etnohistoria}

En julio de 1989 se celebró en la Universidad de Buenos Aires el Primer Congreso Internacional de Etnohistoria con el doble objetivo de reunir a un conjunto internacional de especialistas en etnohistoria andina y de otorgar legitimidad al equipo de investigación que organizó el evento, los miembros de la Sección Etnohistoria (UBA). Esta institución, dirigida por Ana María Lorandi, fue creada en 1985 como consecuencia de la reorganización institucional de la UBA que aconteció tras la vuelta de la democracia en 1983. El primer CIE representó un hito en la conformación de una red académica internacional en torno a la etnohistoria andina, campo que revolucionó la investigación de la región y que continúa manteniendo una impronta interdisciplinar e internacional. Seguidamente nos detendremos en algunas características generales de la organización del primer CIE, para situar la discusión que se dio en relación con el simposio de estrategias en concreto.

\section{La organización de los simposios}

Las primeras referencias al primer CIE datan de mediados de 1987. La Comisión Organizadora -conformada por su presidenta Ana María Lorandi y las secretarias Mercedes del Río y Ana María Prestaenvió las primeras invitaciones a los coordinadores de simposio y, dos meses más tarde, escribieron a John Murra, Gunnar Mendoza y Alberto Salas, quienes serían homenajeados en el evento.

Los simposios propuestos inicialmente fueron "Rebeliones", "Simbolismo y Sincretismo Religioso", "Estrategias adaptativas" y "Esclavitud y Relaciones interétnicas", este último pensado para la asistencia de investigadores de áreas no andinas. A lo largo de las circulares algunos de estos títulos fueron modificados, quedando el listado definitivo de la siguiente manera: "Rebeliones", "Simbolismo y Sincretismo Religioso", "Estrategias de supervivencia", "Pasado y presente en las relaciones interétnicas en el Gran Chaco, Brasil central y Amazonia" y "Relaciones fronterizas y sociedad indígena en los territorios meridionales de Argentina y Chile (siglo XVI a XIX)".

El antropólogo estadounidense Frank Salomon (1946-) fue llamado para encargarse del simposio "Rebeliones". Para el simposio "Simbolismo y Sincretismo Religioso" fue en primer término tentada la historiadora María Rostworowski (1915-2016), pero finalmente tomó su lugar el antropólogo peruano Luis Millones Santagadea (1940-). El coordinador correspondiente al simposio "Estrategias de supervivencia" también tuvo que ser reemplazado. Inicialmente se había convocado al antropólogo británico Tristan Platt (1944-), luego a Xavier Albó (1934-) y Thierry Saignes (1946-1992). Finalmente, fue el historiador francés quien ocupó el puesto. El cuarto simposio, "Pasado y presente en las relaciones interétnicas en el Gran Chaco, Brasil central y Amazonia”, fue coordinado por la antropóloga argentina Alejandra Siffredi (1938-), mientras que el simposio "Relaciones fronterizas y sociedad indígena en los territorios meridionales de Argentina y Chile (siglo 
XVI a XIX)" fue coordinado por el historiador Raúl José Mandrini (1943-2015). Se incorporó también una Sesión de Comunicaciones a cargo del historiador Juan Carlos Garavaglia (1944-2017).

La elección de los coordinadores nos permite apreciar, por una parte, la búsqueda de balance entre investigadores destacados de origen local y extranjero y, por otra, nos ofrece algunas pistas sobre el recorte espacial del primer CIE. Podemos notar que todos los investigadores extranjeros contactados para tareas de coordinación se desempeñaban en el área andina o en sus márgenes. Mientras que los coordinadores locales se especializaban mayormente en áreas no andinas dentro de la Argentina, como Pampa-Patagonia, Chaco y Litoral. ${ }^{4}$

\section{Alcances y límites de "estrategia"}

Si bien los organizadores del congreso plantearon lineamientos generales para los simposios, los cuales referían tanto a las temáticas como a las áreas geográficas y a la temporalidad, el contenido específico de cada uno fue ajustado en diálogo con cada coordinador. Ya en la correspondencia preliminar se hicieron manifiestas algunas discusiones que dan cuenta de los debates que se mantenían en la comunidad de etnohistoriadores. A través de las cartas conservadas en el Archivo Institucional de la Sección Etnohistoria, identificamos en primer lugar de qué manera el alcance del concepto de estrategia se encontraba entre los grandes ejes de discusión que atravesaron la organización del primer CIE. Luego, exploramos los solapamientos que advertían los participantes entre dos de los simposios del Congreso: "Estrategias de supervivencia" y "Rebeliones".

El simposio sobre "estrategias" fue consecuencia lógica de la fuerza que tenía tal temática en la etnohistoria de la década del ochenta. Como hemos visto, esto se dio en el marco mayor de su auge en las ciencias sociales en general y, como en otros campos, en la etnohistoria condujo a una proliferación de investigaciones centradas en el concepto, que implicaron también una discusión sobre sus alcances y sentidos. En el caso del CIE, la discusión se

4 Un análisis en detalle sobre este tema puede encontrarse en Ramos y Chiappe (2018). cristalizó justamente en la correspondencia del simposio "Estrategias de supervivencia". Posteriormente a la aceptación de Saignes para la coordinación, Lorandi le hizo llegar mayores detalles sobre cómo preveía la comisión organizadora el funcionamiento del espacio: "Seguramente, dentro de este simposio, se concentrará la mayor cantidad de exposiciones o trabajos, ya que el espectro será lo suficientemente amplio como para incluir migraciones, demografía, aspectos laborales y control de recursos. Todo ello sujeto a tus sugerencias" (Lorandi, 1988a).

Solo dos semanas después Lorandi recibió la respuesta de Saignes en la que este advertía sobre la necesidad de hacer un recorte para evitar la dispersión temática y poner un límite a la cantidad de ponentes.

El hecho de que dentro del simposio "estrategias" (con un título tan general: ¿qué es lo que no es adaptativo? Hasta las rebeliones pueden serlo a su manera), "se concentrará la mayor cantidad de exposiciones o trabajos" refuerza mi sospecha que se vuelva una especie de catálogo caótico (en el mejor de los casos) sin real coherencia interna, como lo vemos en cualquier reunión de este tipo, situación que personalmente deploro y no deseo reproducir (Saignes, 1988a).

Estos reparos fueron relativizados por Lorandi, recordándole que las ponencias concernientes a "rebeliones" tendrían un simposio exclusivo y asegurándole además que, dentro de la temática general, el coordinador podría realizar las delimitaciones que le parecieran pertinentes.

Estamos de acuerdo contigo que por "estrategias..." se puede entender todo, pero no me parece imposible restringirlas al uso más corriente, es decir, exploración limitada de los mecanismos que ofrece la sociedad colonial. Si bien la rebelión puede ser una estrategia, por su especificidad, muy bien puede ser considerada en bloque con aquellas que directamente han pretendido expulsar al invasor o mantener aislada a la sociedad local como ocurrió con los calchaquíes en el N.O. (Lorandi, 1988b). 
Un "borrador" de agosto de 1988 precisa el alcance final que Saignes otorgó al simposio. Habría dos temas principales: "caciques y agentes mediadores [y] relaciones interétnicas desde las dimensiones demográficas y migratorias" resueltos por dentro de un espacio que incluía el "Área: centro-sur andina y [el] $\mathrm{NO}$ argentino, con sus periferias amazónica, chaqueña y del Pacífico" (Saignes, 1988b).

La evaluación de las ponencias efectivamente presentadas permite comprobar que los reparos de Saignes con respecto a la posible masividad de la convocatoria estuvieron justificados, ya que se presentaron un total de veinticinco trabajos, la reunión más convocante del primer CIE. Con respecto a los ejes propuestos por el coordinador, queda claro que -si bien hubo varias ponencias vinculadas a los temas que este consideraba pertinentes- otras líneas atravesaron el simposio y se relacionaron con las problemáticas territoriales (litigios, desnaturalizaciones), de identidad y mestizaje (lo que en términos actuales podríamos leer en clave de etnogénesis) y, en menor medida, la articulación de la arqueología con la etnohistoria.

La ubicuidad de la temática de estrategias señalada por Saignes páginas atrás puede verse en una carta de 1988 enviada a Lorandi por Erick Langer. Al presentar su resumen de ponencia, titulado: "Las 'guerras chiriguanas'; resistencia y adaptación en la frontera surboliviana (siglo XIX)”, Langer planteó que "habría dos simposios, uno bajo la dirección de Frank Salomon [Rebeliones] y el otro bajo la dirección de Tristan Platt [Estrategias de supervivencia, finalmente a cargo de Saignes] que podrían ser relevantes (si aceptas la ponencia)" (Langer, 1988).

Un caso similar fue el del trabajo de Thomas Abercrombie: 'Emergence of de 'plebe' and 'popular culture' in mid-XVIII th c. Potosí: carnaval, rebellion an the week-end miners". En una carta de Salomon a Lorandi fechada el 4 de enero de 1989, este señaló que, si bien la ponencia estaba programada para "Estrategias de supervivencia", el autor de la misma consideraba que el tema cuadraba mejor con el simposio de Rebeliones (Salomon, 1989).

Assadourian (1988), realizando un puente entre las temáticas de ambos simposios, se preguntaba por la pertinencia de caracterizar como Rebeliones o como Estrategias ciertos fenómenos sociales:

Imagino que el simposio Rebeliones resulta ineliminable por tradición, pero yo sostengo que hay resistencias que no son ni motines, tumultos o rebeliones que provocan efectos estructurales y, por ello, resultan más interesantes que las derrotas para percibir la capacidad histórica de las organizaciones indígenas; no sé si esto se podrá captar en el simposio Estrategias de Supervivencia.

Para entender a qué aludía Assadourian con 'tradición', se debe tomar en cuenta que el estudio de los diferentes levantamientos sociales contra el orden colonial sucedidos en el Perú y el Alto Perú a fines del siglo XVIII había cobrado nuevo impulso desde 1970, en el marco de la agitada política latinoamericana, de la publicación - por parte del gobierno de Perú- de cuantiosa documentación hasta entonces inédita (CNSIP, 1971) y del cumplimiento del bicentenario del levantamiento de Tupac Amaru en 1980. En estos años se multiplicaron también varias obras sobre rebeliones en el Alto Perú. ${ }^{5}$ Todo esto permitió un avance en las investigaciones que habilitó también perspectivas que excedieron los tópicos clásicos.

\section{Estrategias en la Nueva Etnohistoria}

En los apartados precedentes describimos la incorporación del concepto de estrategias en el marco de la Nueva Etnohistoria a través de dos conjuntos de fuentes, publicaciones y correspondencia. Si en un primer momento, previo a los ańos ochenta, el desafío de los etnohistoriadores consistió en instalar como una temática de legítimo interés la historia andina, lo que ocurrió con la Nueva Etnohistoria fue una complejización, necesaria para abordar a través de distintos aspectos (económicos, políticos, simbólicos, etc.) cómo el mundo andino se articuló con el colonial. ${ }^{6}$ Fue esta necesidad de superar un

5 Sobre la historiografía de este tema puede consultarse Serulnikov (2012).

6 Empleamos aquí categorías propias del período manifiestas en las fuentes consultadas. Justamente las investigaciones de la Nueva Etnohistoria contribuyeron a una 
tratamiento dicotómico lo que llevó a que un concepto como el de estrategias resultara atractivo para los etnohistoriadores.

Se intentaba responder básicamente dos preguntas: ¿Cómo pensar el concepto de estrategias en el marco de la etnohistoria?, y ¿qué fenómenos sociales pueden ser entendidos en términos de estrategias?

Existió un acuerdo de época sobre la validez del concepto a la hora de tratar de manera articulada la sociedad colonial y -en particular- fenómenos colectivos de cierta duración. Sin embargo, diferente cosa fue pactar cuál era el conjunto de prácticas que podían válidamente incluirse dentro del concepto. Esto generó un intenso debate, no solo por la ambigüedad del mismo, sino también porque tuvo que enfrentarse al carácter muchas veces arbitrario de las segmentaciones temáticas. En efecto, la compartimentalización de las investigaciones sobre la sociedad andina colonial no obedecía exclusivamente a una delimitación conceptual de las prácticas bajo estudio, sino también a coyunturas históricas que instalaron determinados temas en el debate académico, y que fueron cobrando preeminencia hasta el punto de convocar a un conjunto de especialistas. Piénsese que 'rebeliones' - un tema dentro del universo de las sociedades andinas- adquirió una notoria independencia más allá de que conceptualmente pudiera o no ser este entendido en términos de estrategias. Puntualmente, estamos aquí haciendo referencia a que el bicentenario de la rebelión de Tupac Amaru y la tracción que este provocó con respecto a la publicación de fuentes documentales asociadas constituyó el marco para que el tópico de rebeliones -más allá del Perú y el Alto Perú, como puede verse en las presentaciones al simposio del primer CIE (Ramos y Chiappe, 2018) - se instalara con fuerza en la agen$\mathrm{da}$ académica de fines de la década.

A partir de los materiales concretos sobre la Nueva Etnohistoria que hemos tratado podemos afirmar que, en las compilaciones temáticas (Harris et al., 1987; Moreno Yáńez y Salomon, 1991; Stern 1990a

atomización de lo andino que, sumada a la posterior crítica a las categorías totalizadoras que se produjo en los noventa, cuestionaron la denominación de mundo andino y otras adjetivaciones similares (Ramos, 2016a).
[1987]), el concepto se utilizó para complejizar las investigaciones sobre temas ya instalados, tales como resistencia, formas de intercambio y cambio/ continuidad social. Esto es, se trató de marcar los avances puntuales en la implementación del concepto a través de casos concretos de investigación. Los documentos inéditos del congreso, en cambio, nos habilitaron la posibilidad de acceder a las discusiones del momento sobre su ambigüedad y su solapamiento de sentido. Este acceso privilegiado a un material poco frecuente permitió reponer diálogos más generales, no atados a una investigación puntual. Los intercambios refieren particularmente al alcance del concepto y a cómo hacerlo operativo en los términos del campo disciplinar.

Como vimos, el concepto de estrategias -vía privilegiada para el estudio de las relaciones sociales que se dieron en el escenario colonial andino- apareció con fuerza en la década del ochenta a través de diversos medios de comunicación del campo etnohistórico. En nuestra consideración, el hecho de que el mismo haya tenido un impacto tan profundo en la etnohistoria se debe a que, al permitir recuperar y articular las experiencias pasadas y la innovación puestas en práctica, resultó particularmente operativo para los estudios realizados en un campo que ha sido definido por el carácter bifronte de sus investigaciones. En efecto, estas se han focalizado tanto en los logros andinos y en las formas de adaptación y reestructuración que los pueblos originarios implementaron antes y después de la Conquista, como también en la desestructuración que las mismas poblaciones experimentaron bajo la expansión colonial (Chiappe, 2018). Este interés se ha mantenido en la etnohistoria a lo largo de todo su desarrollo bajo diferentes fórmulas: cambio/continuidad, adaptación/resistencia, y etnogénesis/etnificación (Ramos, 2016). Entendemos que es por ello que el concepto de estrategias continúa jugando un rol central en las investigaciones actuales.

\section{Consideraciones finales}

En este artículo establecimos que, a través de la aplicación del concepto de estrategias en las ciencias sociales, se buscó recuperar la agencia de los sujetos y 
aprehender los cambios y continuidades sociohistóricos. Dimos cuenta de cómo, en un campo disciplinar específico, se replicaron ciertas problemáticas comunes a otros campos del conocimiento, y de qué modo también aparecieron dificultades, propias de ese campo de investigación.

Argumentamos que el intercambio epistolar constituye un insumo particularmente rico para acceder a las reflexiones exploratorias, no acabadas, de los investigadores, las cuales -más que respuestas definitivas- dejan preguntas abiertas. Así pensado, este tipo de materiales permite tomar distancia del ideal corriente sobre el conocimiento científico como cerrado o acabado, y acceder a las formas que adquiere la ciencia en ejecución, a las prácticas que -en definitiva- la constituyen. Es por ello que, a la hora de encarar investigaciones sobre historia conceptual o de las ideas, creemos de importancia utilizar fuentes de información complementarias y de diferente tipo.

Finalmente, la revisión hecha del uso del concepto de estrategias, en un momento específico y en un campo particular, puede capitalizarse al situarla en el marco general de las ciencias sociales. En la actualidad, a pesar del empleo extendido del concepto, Viazzo y Lynch (2002, p. 425) consideran que este permanece ambiguo y mal definido.

We can legitimately wonder whether we are dealing here with a well-developed concept or just with a fashionable and rather equivocal term. A certain amount of polysemy can of course be expected when a term is used -as is the case with 'strategy'- across the social sciences, from economics to sociobiology. However, even if we restrict our attention to family history and to social anthropology, we immediately notice an alarming degree of looseness and confusion.

La referencia tan extendida al concepto, que condujo a que en cada campo y disciplina se le asignase sentido en relación con otros conceptos propios de esos espacios, contribuyó a empleos dispares, contradictorios, o al menos no compatibles entre sí.

Aunque no ocupe ya un lugar central en los debates y reflexiones, el concepto de estrategia se encuentra plenamente vigente en la ciencias sociales. Consideramos que esta vigencia sin centralidad se debe a que se encuentra en gran medida naturalizado. Es decir, en términos generales, se da por sentado su sentido y se lo emplea como una categoría transparente. Por supuesto, este fenómeno no es exclusivo ya que alcanza también a otros conceptos, como por ejemplo el de proceso (veáse Gaztańaga, 2014).

El estudio de los mecanismos a través de los cuales se dirimen los alcances de los conceptos y la sistematización de sus sentidos constituyen una herramienta del saber reflexivo de las condiciones y de los límites sociales de la práctica científica. El conocimiento así generado no constituye mera entelequia, todo lo contrario, ya que lleva a reforzar, mediante la toma de conciencia y la vigilancia epistemológica que favorece, la capacidad de conocer científicamente la realidad (Bourdieu, 2008 [1984]).

\section{Referencias citadas}

Abercrombie, T. (1991). Articulación y etnogénesis. En Moreno, S. y Salomon, F. (Comps.). Reproducción y transformación de las sociedades andinas siglos XVI-XX (pp. 197-212). Quito: Abya-Yala.

Albó, X. (1990 [1987]). De MNRistas a Kataristas a Katari. En Stern, S. (Comp.). Resistencia, rebelión y conciencia campesina en los Andes, siglos XVIII al XX (pp. 312-363). Lima: IEP.

Argüello, O. (1981). Estrategias de supervivencia, un concepto en busca de su contenido. Demografía y economía, XV(2), 190-202.

Assadourian, S. (1988, Mayo 2). Carta a Ana María Lorandi. Archivo Institucional Sección Etnohistoria (Colección ICIE), Universidad de Buenos Aires, Buenos Aires.

Berry, B. (1967). Strategies, Models, and Economic Theories of Development in Rural Region. Agricultural Economic Report, 127. Washington, DC: Economic Research Service.

Bourdieu, P. (1972). Les stratégies matrimoniales dans le système de reproduction. Annales ESC, 27, 1105-1127.

Bourdieu, P. (1988). La Distinción. Criterios y bases sociales del gusto. Madrid: Taurus. 
Bourdieu, P. (2007 [1980]). El sentido práctico. Buenos Aires: Siglo XXI.

Bourdieu, P. (2008 [1984]). Homo Academicus. Buenos Aires: Siglo XXI.

Campbell, L. (1990 [1987]). Ideología y faccionalismo durante la gran rebelión, 1780-1782. En Stern, S. (Comp.). Resistencia, rebelión y conciencia campesina en los Andes, siglos XVIII al XX (pp. 118-139). Lima: IEP.

Chiappe, C. (2018). Entre las luchas pasadas y las presentes. Antecedentes, surgimiento y desarrollo de la etnohistoria andina chilena (Tesis de Doctorado en Antropología). Universidad de Buenos Aires, Buenos Aires.

Comisión Nacional del Sesquicentenario de la Independencia del Perú. (1971). La rebelión de Tupac Amaru. Lima: CNSIP.

Consejo de Dirección Prismas. (2007). Presentación. Prismas. Revista de historia intelectual, 11, 151.

Dandler, J. (1987). Diversificación, procesos de tabajo y movilidad espacial en los Valles y Serranías de Cochabamba. En Harris, O., Larson, B. y Tandeter, E. (Comps.). La participación indigena en los mercados surandinos (pp. 639-682). La Paz: Ceres.

Gaztañaga, J. (2014). El proceso como dilema teórico y metodológico en antropología y etnografía. PUBLICAR en antropología y ciencias sociales, 12(16), 35-58.

Gutiérrez, A. (2002). Problematización de la pobreza urbana tras las categorías de Pierre Bourdieu. Cuadernos de Antropología Social, 15, 9-27.

Harris, O., Larson, B. y Tandeter, E. (1987). Antecedentes. En Harris, O., Larson, B. y Tandeter, E. (Comps.). La participación indígena en los mercados surandinos (pp. 1941). La Paz: Ceres.

Hidalgo Lehuedé, J. (2004). Etnohistoria e interdisciplinariedad en Chile. Desde sus orígenes hasta 1980. En Historia andina en Chile (pp. 655-684). Santiago: Universitaria.

Kirch, P. (1980). The Archaeological Study of Adaptation: Theoretical and Methodological Issues. En Schiffer, M. (Ed.). Advances in Archaeological Method and Theory, vol. 3 (pp. 101-156). Nueva York: Academic Press.
Langer, E. (1988, Julio 19). Carta a Ana María Lorandi. Archivo Institucional Sección Etnohistoria (Colección ICIE), Universidad de Buenos Aires, Buenos Aires.

Lorandi, A. (1988a, Abril 20). [Carta a Thierry Saignes]. Archivo Institucional Sección Etnohistoria (Colección ICIE), Universidad de Buenos Aires, Buenos Aires.

Lorandi, A. (1988b, Mayo 12). [Carta a Thierry Saignes]. Archivo Institucional Sección Etnohistoria (Colección ICIE), Universidad de Buenos Aires, Buenos Aires.

Monereo, C. (1999) (Coord.). Estrategias de enseñanza y aprendizaje. Barcelona: Graó.

Moreno Yáñez, S. y Salomon, F. (1991). Reproducción y transformación de las sociedades andinas, siglos XVI-XX. Quito: Abya-Yala.

Morrone, A. (2007). El liderazgo étnico como problema tempranocolonial. La situación de Pacajes en los siglos XVIXVII (Tesis de Licenciatura en Historia). Universidad de Buenos Aires, Buenos Aires.

Pease, F. (1987 [1974]). Etnohistoria andina, problemas de fuentes y metodología. En Pérez Zevallos, J. y Pérez Gollán, J. (Eds.). La etnohistoria en Mesoamérica y los Andes (pp. 209-228). México, DF: INAH.

Pérez Zevallos, J. y Pérez Gollán, J. (1998). Introducción. En Actas del IV Congreso Internacional de Etnohistoria (p. 14). Lima: Pontificia Universidad Católica del Perú.

Platt, T. (1990 [1987]). La experiencia andina de liberalismo boliviano entre 1825 y 1900: raíces de la Rebelión de Chayanta (Potosí) durante el siglo XIX. En Stern, S. (Comp.). Resistencia, rebelión y conciencia campesina en los Andes, siglos XVIII al XX (pp. 261-306). Lima: IEP.

Ramos, A. (2016a). El desarrollo de la Etnohistoria andina como campo interdisciplinar: interacciones entre Historia, Arqueología y Antropología (Perú, Bolivia y Argentina, 1970-2005) (Tesis de Doctorado en Antropología). Universidad de Buenos Aires, Buenos Aires.

Ramos, A. (2016b). Etnohistoria(s): contextos de emergencia y vigencia discutida. Relaciones de la Sociedad Argentina de Antropología, 41(1), 15-34.

Ramos, A. (2018). El desarrollo de la Etnohistoria andina a través de la (re)definición de lo andino (1970-2005). Fronteras de la Historia, 23(2), 8-43. 
Ramos, A. y Chiappe, C. (2018). En la trama de la etnohistoria americana. Tarija, Bolivia: La pluma del escribano.

Ridley, M. (1993). Evolution. Oxford, UK: Blackwell Scientific Publications.

Rivera, S. (1984). Oprimidos pero no vencidos: luchas del campesinado aymara y qhechwa de Bolivia, 1900-1980. La Paz: HISBOL.

Saignes, T. (1987). Ayllus, mercado y coacción colonial: el reto de las migraciones internas en Charcas (siglo XVII). En Harris, O., Larson, B. y Tandeter, E. (Comps.). La participación indigena en los mercados surandinos (pp. 111158). La Paz: Ceres.

Saignes, T. (1988a, Mayo 4). Carta a Ana María Lorandi. Archivo Institucional Etnohistoria (Colección ICIE), Universidad de Buenos Aires, Buenos Aires.

Saignes, T. (1988b, Agosto 18). Borrador carta a expositores. Archivo Institucional Sección Etnohistoria (Colección ICIE), Universidad de Buenos Aires, Buenos Aires.

Salomon, F. (1989, Enero 4). Carta a Ana María Lorandi. Archivo Institucional Sección Etnohistoria (Colección ICIE), Universidad de Buenos Aires, Argentina.

Salomon, F. (1990 [1987]). Culto a los ancestros y resistencia frente al Estado en Arequipa entre los años 1748 y 1754. En Stern, S. (Comp.). Resistencia, rebelión y conciencia campesina en los Andes, siglos XVIII al XX (pp. 144163). Lima: IEP.

Salomon, F. (1991). Tres enfoques cardinales en los actuales estudios andinos. En S. Moreno y F. Salomon (Comps.), Reproducción y transformación de las sociedades andinas siglos XVI-XX (pp. 7-26). Quito: Abya-Yala.

Sánchez Parga, J. (1984). Estrategias de supervivencia. En Sánchez Parga, J., Chiriboga, M., Ramón, G., Guerrero, A., Durston, J. y Crivelli, A. (Comps.). Estrategias de supervivencia en la comunidad andina (pp. 9-58). Quito: Centro Andino de Acción Popular.
Serulnikov, S. (2012). La Insurrección Tupamarista: Historias e Historiografías. 20/10 Historia, 1-12.

Stern, S. (Comp.). (1990a [1987]). Resistencia, rebelión y conciencia campesina en los Andes, siglos XVIII al XX. Lima: IEP.

Stern, S. (1990b [1987]). Prefacio. En Stern, S. (Comp.). Resistencia, rebelión y conciencia campesina en los Andes, siglos XVIII al XX (pp. 13-16). Lima: IEP.

Stern, S. (1990c [1987]). Nuevas aproximaciones al estudio de la conciencia y las rebeliones campesinas: las implicancias de la experiencia andina. En Stern, S. (Comp.). Resistencia, rebelión y conciencia campesina en los Andes, siglos XVIII al XX (pp. 25-41). Lima: IEP.

Stern, S. (1990d [1987]). La era de la insurrección andina, 1742-1782: una reinterpretación. En Stern, S. (Comp.). Resistencia, rebelión y conciencia campesina en los Andes, siglos XVIII al XX (pp. 49-96). Lima: IEP.

Szemiński, J. (1990 [1987]). ¿Por qué matar a los españoles? Nuevas perspectivas sobre la ideología andina de la insurrección en el siglo XVIII. En S. Stern, S. (Comp.). Resistencia, rebelión y conciencia campesina en los Andes, siglos XVIII al XX (pp. 164-186). Lima: IEP.

Urton, G. (1991). Las unidades de análisis en el estudio de la reproducción y transformación de las sociedades andinas. En Moreno-Yáñez, S. y Salomon, F. (Comps.). Reproducción y transformación de las sociedades andinas, siglos XVI-XX (pp. 29-46). Quito: Abya-Yala.

Viazzo, P. y Lynch, K. (2002). Anthropology, Family History, and the Concept of Strategy. IRSH, 47, 423-452.

Von Neumann, J. y Morgenstern, O. (2007 [1944]). Theory of Games and Economic Behavior. Princeton, NJ: Princeton University Press.

Wachtel, N. (1971). La vision des vaincus: les indiens du Pérou devant la conquête espagnole, 1530-1570. Paris: Gallimard. 